photograph the same part of the sky weeks or months apart. They then look for objects that shift position between the two images, relative to the background stars. The team first spotted most of the new Jovian moons using the Blanco 4-metre telescope at the Cerro Tololo Inter-American Observatory in Chile, and followed up with further observations at other telescopes.

\section{STRANGE SATELLITES}

All the newfound moons are small, between about 1 and 3 kilometres across. Seven of them travel in remote orbits more than 20 million kilometres away from Jupiter, and in the opposite direction from the planet's rotation. That puts them in the category known as retrograde moons.

The eighth moon stands out because it travels in the same region of space as the retrograde moons, but in the opposite direction (that is, in the same direction as Jupiter's spin). Its orbit is also tilted with respect to those of the retrograde moons. That means it could easily smash into the retrograde moons, pulverizing itself into oblivion. It may be the leftovers of a bigger cosmic collision of this nature in the past, Sheppard says.
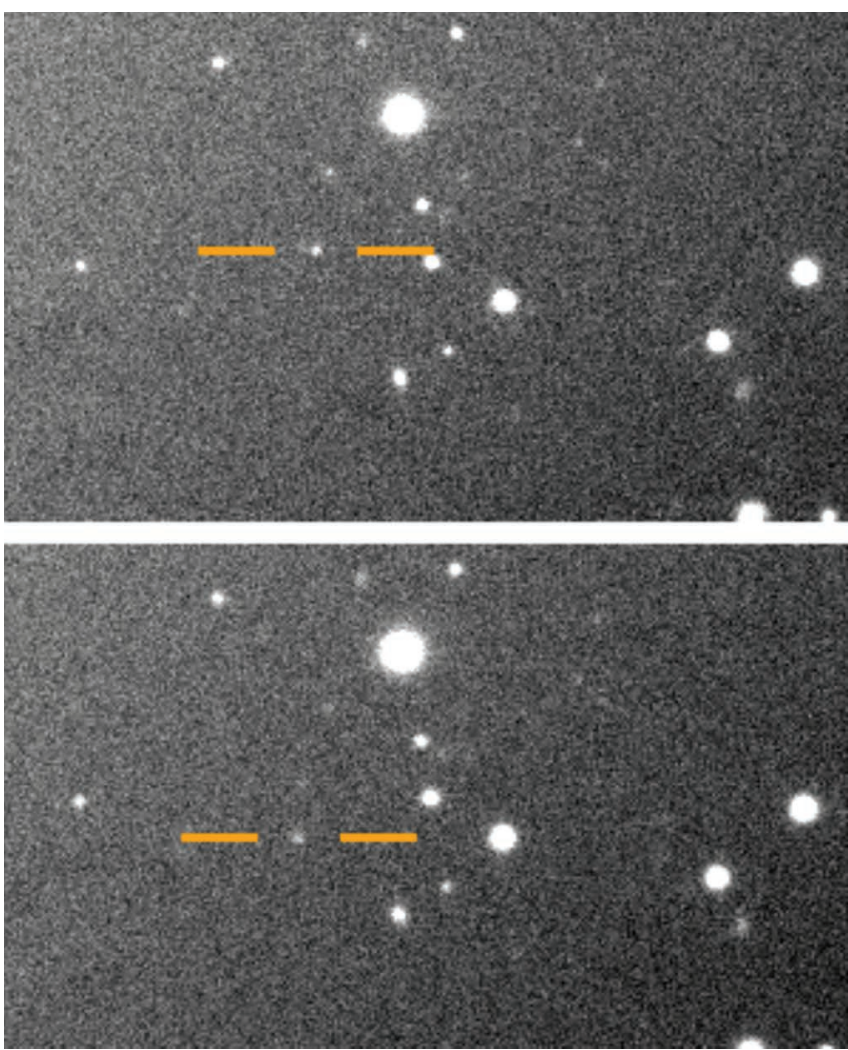

These images show the movement of the Jovian moon dubbed Valetudo (labelled in yellow) relative to the background stars.

Jupiter's moons are named after gods with connections to the mythological deities Jupiter or Zeus. Sheppard has proposed naming the oddball Valetudo, after one of Jupiter's descendants, the Roman goddess of hygiene and health.

The ninth and tenth newfound moons orbit closer to Jupiter, moving in the same direction as the planet.

Had all these small moons formed at the same time as Jupiter, they probably would have been captured by the gas and dust still swirling around the newborn planet, and have been engulfed. Their existence suggests that they are leftovers of later collisions between space rocks that left the debris encircling Jupiter.

If astronomers can work out the history of these collisions, they could also determine the sizes of any satellites that were pulled into the orbit of a young Jupiter. "That's the big question, and that's what makes these ten new moons interesting," says Douglas Hamilton, an astronomer at the University of Maryland in College Park. "How can we link all this to how planets formed?"

Sheppard says there might still be a few more moons of Jupiter to discover - as yet unseen because they were hiding in the Sun's glare when the scientists were looking. Saturn, the runnerup to Jupiter in the moon competition, has 62 known satellites.

\title{
Gene therapy in mouse fetuses treats deadly disease
}

\section{The method could minimize damage from disease if a condition is diagnosed in utero.}

\section{BY HEIDI LEDFORD}

G ene therapy administered in the womb could be used to treat a deadly genetic disease, a study in fetal mice suggests.

The results could add to the increasingly popular approach of using prenatal gene therapy to minimize the damage wrought by some genetic diseases. The US Food and Drug Administration approved the first gene therapy for adults and children last year, and more treatments are crowding pharmaceutical pipelines around the world.

Simon Waddington, the lead author of the latest study, says he used to meet with shocked stares when he talked about treating fetuses with gene therapy. "It had gotten to the point where I'd given up on telling people that fetal gene therapy is a good idea," says Waddington, who studies gene therapy at University College London. "And now, not infrequently, people turn to me and say, 'You know what would be a good idea? Fetal gene therapy."

The mouse study, published on 16 July in Nature Medicine ${ }^{1}$, uses prenatal gene therapy to tackle a condition - acute neuronopathic Gaucher's disease - caused by mutations in a gene called GBA. These mutations disrupt the breakdown of a particular fatty molecule, or lipid. As a result, the lipid accumulates in brain cells and other parts of the body, contributing to organ dysfunction.
The study looks at whether the disease can be treated by using a virus to supply normal copies of GBA to a developing fetus. That could minimize the irreparable brain damage that arises as the lipid accumulates.

Some forms of Gaucher's disease can be treated by supplying normal copies of the GBA enzyme to break down lipids, but that enzyme cannot cross from the blood into the brain. Children with acute neuronopathic Gaucher's disease rarely live past two years.

\section{DIFFICULT CROSSING}

The condition is so devastating that colleagues were sceptical about his team's ability to treat it, says Waddington. "People told me, 
> 'You're not going to touch this."'

One hurdle was simply getting the virus to carry the healthy gene into the brain. Viruses used in previous tests had to be injected directly into the brain, and then they diffused only a short distance from the injection site. But in 2009, researchers showed $d^{2,3}$ that a particular virus, simply injected into the blood, could reach the central nervous system. From there, it dispersed throughout the brain.

Waddington began working with mice, loading up the virus with a normal copy of $G B A$ and looking for ways to express it specifically in the central nervous system. His team tested its virus in fetal mice carrying $G B A$ mutations that cause symptoms similar to neuronopathic Gaucher's disease. Such mice normally live for only 15 days after birth; treated mice, however, survived for at least 18 weeks and were able to move about normally.

\section{FETAL FRONTIER}

The work is impressive, says Tippi MacKenzie, a fetal-medicine specialist at the University of California, San Francisco. MacKenzie has been conducting a clinical trial of prenatal stem-cell transplants. "Fetal gene therapy or enzyme-replacement therapy may be the next frontier," she says. "It is wonderful to see this kind of rigorous research, to take us one step further."

Treating fetuses has several potential advantages. Chief among them is the potential to minimize the damage caused by a genetic disease. Some of these conditions - such as neuronopathic Gaucher's disease and spinal muscular atrophy - can cause irreversible symptoms before birth.

It is also easier to administer some therapies to the brain in a developing fetus than

"People told me, 'You're not going to touch this." in an adult or child, because the bloodbrain barrier - a membrane that prevents some molecules from crossing into the brain from the blood - is more permeable.

"Even one day after birth, it's harder to get into the brain," says Jerry Chan, an obstetrician and gynaecologist at Duke-NUS Medical School in Singapore.

And the fetal immune system is also still developing, making it less likely to recognize the newly expressed protein as foreign. Adult immune systems sometimes generate antibodies against the new protein, which can prevent it from carrying out its function.

\section{WEIGHING THE RISKS}

Chan and others have previously tested fetal gene therapy to treat haemophilia in mice and macaques, and Chan expects that there will be interest in doing so for several metabolic diseases similar to Gaucher's.

But there are risks. Researchers developing a prenatal gene therapy must think not only about the fetus, but also about the mother, who will inevitably receive a dose of treatment as well, says Chan.

And clinicians have to be absolutely certain that the mutation they've found will cause disease, notes Waddington. This may mean combining genetic tests with other tests performed in utero, to confirm the disorder.

"We're now at the point where it's possible to diagnose these diseases," he says. "It's making people think: maybe we should be doing this."

1. Massaro, G. et al. Nature Med. https://doi. org/10.1038/s41591-018-0106-7 (2018)

2. Foust, K. D. et al. Nature Biotechnol. 27, 59-65 (2009).

3. Duque, S. et al. Mol. Ther. 17, 1187-1196 (2009).

\section{CORRECTION}

The News Feature 'Bat man' (Nature 559, $165-168 ; 2018$ ) talked of the Egyptian fruit bats under study being collected in the Jordanian hills instead of the Judean hills. It also incorrectly labelled the picture of a neural logger as being a GPS logger. 